\title{
Neuromédiateurs bronchiques et pulmonaires
}

Une bonne connaissance de l'innervation bronchique et pulmonaire est indispensable à la compréhension de la physiopathologie et au traitement de la bronchoconstriction des asthmatiques. Outre les systèmes cholinergiques et sympathiques, il semble que des systèmes peptidergiques dont les médiateurs sont, entre autres, le vasoactive intestinal peptide (VIP), le calcitonin gene related peptide (CGRP) ou la substance $P$, jouent un rôle important dans le contrôle du tonus bronchique et de la perméabilité capillaire.

Béatrice M. Grandordy Chef de clinique-assistant des hôpitaux

\section{ADRESSE}

B.M. Grandordy : service de pneumologie, hôpital Cochin, 27, rue du Faubourg SaintJacques, 75014 Paris, France.

\section{TIRÉS A PART}

B.M. Grandordy : service de pneumologie, hôpital Cochin, 27, rue du Faubourg SaintJacques, 75014 Paris, France.

$\mathrm{m} / \mathrm{s} n^{\circ} 1$ vol. 4, janvier 88

permis récemment d'améliorer la

1 existe dans toutes les espèces une innervation pulmonaire et bronchique abondante, variable avec l'espèce, dont les terminaisons nerveuses sensitives (voies afférentes) et motrices (voies efférentes) peuvent libérer des neuromédiateurs [1]. Les terminaisons sensitives peuvent jouer le rôle de récepteurs à différents stimuli, mécaniques, chimiques ou physiques, et libérer des peptides, qui à leur tour seront actifs sur différentes cellules pulmonaires. Les voies efférentes innervent le muscle lisse, les glandes sécrétrices et les vaisseaux ; différents neuromédiateurs, libérés dans les synapses entre les fibres nerveuses et le tissu effecteur déterminent la bronchoconstriction et/ou la relaxation, la production de mucus et la perméabilité vasculaire.

Les anomalies de fonctionnement du système nerveux autonome, que constitue l'hyper-réactivité des voies aériennes, se manifestent par une bronchoconstriction exagérée, des sécrétions et une réponse vasomotrice anormalement importantes. Les anomalies qui constituent le substratum de l'hyperréactivité pourraient être situées à différents niveaux des voies aériennes, centrales ou périphériques. De nouvelles techniques, comme l'autoradiographie, l'immunocytochimie, la stimulation électrique des voies nerveuses ont connaissance des neuromédiateurs présents dans les poumons et les bronches.

\section{Voies afférentes et récepteurs sensitifs}

La plupart des fibres afférentes sont incluses dans le nerf vague, mais des fibres adrénergiques afférentes, plus rares, ont été décrites dans certaines espèces [2]. L'innervation afférente du poumon humain, peu abondante, est mal connue. Trois types de fibres afférentes sont décrits. Certaines cellules neuroendocrines épithéliales jouent aussi le rôle de neurorécepteurs.

Les récepteurs à l'étirement et les voies de conduction. Les récepteurs à l'étirement sont situés sur les cellules musculaires lisses, y compris dans le poumon humain, et possèdent les propriétés ultrastructurales des mécanorécepteurs. Ils sont stimulés par les modifications de tension de la paroi des voies aériennes.

Ils correspondent à des fibres nerveuses myélinisées. Récepteurs et fibres sont d'adaptation lente. Ils sont responsables de bronchodilatation réflexe, par inhibition du tonus vagal, et également du réflexe de Hering-Breuer, qui inhibe l'activité inspiratoire soutenue. A volume élevé, leur stimulation prolonge l'expiration. Leur rôle chez l'homme n'est pas élu- 


\section{RÉFÉRENCES}

1. Boushey HA, Holtzman MJ, Sheller JR, Nadel JA. Bronchial hyperreactivity. Am Rev Respir Dis 1980 ; 121 : 389-413.

2. Nadel JA. Autonomic regulation of airway smooth muscle. In : Lenfant C, ed. Physiology and Phamacology of the airways. Vol 15: Lung biology in health and disease. New York : Marcel Dekker, 1980 ; 217-39.

3. Lundberg JM, Hökfelt T, Martling CR, Saria A Cuello C. Substance P. immunoreactive sensory nerves in the lower respiratory tract of various mammals including man. Cell Tissue Res 1984 ; 235 : 251-61.

4. Partanen M, Laitinen A, Pelto-Huikko M, Laitinen LA, Hernonen A. Electron microscopic study on granule containing (APUD) cells in adult human lung. Cell Tissue Res 1986 (sous presse).

5. Partanen M, Laitinen A, Hervonen A, Toivanen $A$, Laitinen LA. Catecholamine and acetylcholinesterase containing nerves in human lower respiratory tract. Histochemistry 1982 ; $76: 175-88$.

6. Skoogh BE. Airway ganglia. Clin Resp Physiol 1986 (sous presse).

7. Barnes PJ, Basbaum CB, Nadel JA. Autoradiographic localization of autonomic receptors in airway smooth muscle : marked differences between large and small airways. $A m$ Rev Respir Dis 1983 ; 127 : 758-62.

8. Baron CB, Cunningham M, Strauss JF, Coburn RF. Pharmacomechanical coupling in smooth muscle may involve phosphatidylinositol metabolism. Proc Natl Acad Sci USA 1984 ; 81 : 6899-903.

9. Grandordy BM, Cuss FMC, Sampson AS, Palmer JBD, Barnes PJ. Phosphatidylinositol response to cholinergic agonists in airway smooth muscle : relationship to contraction and muscarinic receptor occupancy. $J$ Phamecol Exp Ther 1986 ; 238 : 273-9.

10. Hashimoto $T$, Hirata $M$, Ito $Y$. A role for inositol 1, 4, 5-triphosphate in the initiation of agonist-induced contractions of dog tracheal smooth muscle. Br J Pharmacol 1985 ; cidé. Il est possible qu'ils soient responsables de la bronchodilatation réflexe lors de l'augmentation de volume pulmonaire qui suit la bronchoconstriction provoquée. Il est aussi possible qu'ils jouent un rôle chez les bronchopathes chroniques, qui respirent à volume élevé, et ils pourraient prolonger l'expiration et raccourcir la durée de l'inspiration chez ces patients. Les récepteurs à l'irritation. Ces récepteurs sont présents dans le larynx, la trachée et le parenchyme pulmonaire. Ils sont situés sous l'épithélium et entre les cellules épithéliales, dans différentes espèces, dont l'homme. Ce sont des mécano- et des chémorécepteurs.

Ils correspondent à des fibres nerveuses myélinisées, d'adaptation rapide, et déchargent lorsqu'ils sont stimulés par ces agents mécaniques (diminution du volume pulmonaire à l'expiration), chimiques (tabac, $\mathrm{SO}_{2}, \mathrm{O}_{3}$ ), et inflammatoires (histamine, sérotonine, prostaglandine F2- $\alpha$ ). Il est également possible qu'ils soient stimulés par la bronchoconstriction elle-même. Ils sont probablement impliqués dans la genèse de la toux, et leur stimulation entraîne une respiration rapide et superficielle, due à une diminution des temps inspiratoire et expiratoire. Les récepteurs des fibres $C$. Ces récepteurs sont situés dans l'épithélium des voies aériennes ; chez l'homme, ces récepteurs se trouvent près de la membrane basale ou de la lumière des grosses voies aériennes. Les terminaisons des fibres $C$ contiennent des neuropeptides. Leur libération se produit lors de la stimulation par la capsaïcine (extraite du piment rouge), l'histamine, certaines prostaglandines et la bradykinine. Il est possible que cette libération de neuropeptides se produise chez l'asthmatique sous l'effet des médiateurs de l'inflammation. Les neuropeptides libérés pourraient alors causer par eux-mêmes une bronchoconstriction, et l'ensemble du système fonctionnerait comme un réflexe d'axone [3], c'est-à-dire un mode de conduction nerveuse où la voie sensitive stimulée trans- met l'information aux branches collatérales sensitives qui vont libérer les médiateurs qu'elles contiennent, sans que l'information ne passe par le système nerveux central.

Les fibres sont à vitesse de conduction lente et, dans certaines espèces, sont trois fois plus nombreuses que les fibres myélinisées. Leur stimulation cause une bronchoconstriction réflexe et une hyperpnée, comme la stimulation des récepteurs à l'irritation. Elle peut aussi conduire à un accroissement de la production de mucus. Il existe, dans la paroi alvéolaire, au contact des capillaires, des fibres $\mathrm{C}$ dont la stimulation conduit à une respiration rapide et superficielle.

Les cellules neuroendocrines. Des cellules, dont la structure histologique est celle des cellules neuroendocrines, sont rencontrées dans l'épithélium bronchique, y compris chez l'homme, au voisinage des nerfs afférents. Ces cellules peuvent libérer de la sérotonine et différents neuropeptides (bombésine, calcitonin gene related peptide, calcitonine, katacalcine, etc.) et il est possible qu'ils activent les fibres nerveuses de voisinage. Les stimuli des cellules neuroendocrines ne sont pas connus, mais il est établi que ces cellules sont sensibles à l'hypoxie, et sont diminuées dans le syndrome de détresse respiratoire du nouveau-né [4].

\section{Neuromédiateurs cholinergiques}

Innervation. Le système cholinergique efférent est abondant dans toutes les espèces, et innerve le muscle lisse ainsi que les glandes, mais pas l'épithélium, les vaisseaux et la paroi alvéolaire. La densité de l'innervation diminue de la trachée aux bronchioles. Les fibres pré- et post-ganglionnaires font synapse dans le ganglion, qui est situé dans la paroi des voies aériennes. En microscopie électronique, les fibres cholinergiques typiques portent des petites vésicules agranulaires contenant l'acétylcholine. Elle est libérée 
dans la synapse neuro-musculaire par la stimulation des fibres cholinergiques [5].

La stimulation cholinergique est responsable d'une bronchoconstriction d'installation rapide, due à la contraction du muscle lisse des voies aériennes de moyen calibre. Il existe un tonus vagal de repos de faible intensité, puisque chez l'homme normal l'atropine est bronchodilatatrice, et que l'inhalation d'anticholinestérase, l'enzyme qui dégrade l'acétylcholine dans la synapse in vivo, cause une bronchoconstriction. Les fibres post-ganglionnaires, présentes dans la bronche humaine, peuvent être stimulées par un champ électrique. La réponse contractile et l'accroissement de la sécrétion de mucus ainsi produits sont bloqués par l'atropine et la tétrodotoxine, une substance bloquant la transmission nerveuse, confirmant que la contraction est due à la présence de fibres postganglionnaires dans la paroi bronchique, et non à la stimulation directe du muscle lisse.

La fonction cholinergique peut être modulée par différents neurotransmetteurs et médiateurs de l'inflammation. La noradrénaline réduit la contraction cholinergique, à des concentrations qui ont peu d'effet sur la réponse musculaire propre à l'acétylcholine, suggérant une inhibition de la libération d'acétylcholine dans la synapse neuro-musculaire, et un contrôle nerveux et non pas musculaire de la voie cholinergique par le système adrénergique. Des récepteurs $\beta$-adrénergiques ont d'ailleurs été mis en évidence dans le ganglion parasympathique. La prostaglandine E2 inhibe aussi la transmission cholinergique chez le chien, alors que la sérotonine la potentialise, suggérant la présence de récepteurs à ces agonistes sur les voies nerveuses cholinergiques. Chez l'homme, la modulation pré-synaptique du système cholinergique est mal connue parce qu'il est difficile de mesurer la libération d'acétylcholine par les nerfs des voies aériennes. Mais il est vraisemblable que certains neuromédiateurs comme les neuropeptides et les médiateurs de l'inflammation peuvent moduler le tonus cholinergique, par l'intermédiaire de récepteurs spécifiques situés sur les voies nerveuses ou les ganglions.

Les ganglions parasympathiques. Les ganglions parasympathiques sont situés dans la paroi des voies aériennes, en général à l'extérieur du muscle lisse, parfois sous l'épithélium. Dans le poumon humain les ganglions sont constitués de 20 corps cellulaires environ. Certaines cellules, de caractère adrénergique, contiennent de grosses vésicules à grains denses et peuvent contenir des catécholamines ou des peptides, suggérant la possibilité d'un contrôle du système cholinergique par le système adrénergique ou nonadrénergique non-cholinergique [6]. Il est possible que différents médiateurs modulent également la fonction cholinergique au niveau ganglionnaire. Des mastocytes sont présents, en contact étroit avec le ganglion. Le rôle du ganglion est mal connu. Il est possible que les ganglions cholinergiques reçoivent des fibres afférentes ; le ganglion participerait alors à un réflexe à point de départ local. Les affections virales chez le cobaye induisent une hyperréactivité, peu sensible à l'atropine mais inhibée par l'hexaméthonium, suggérant qu'un mécanisme nerveux est impliqué.

Les récepteurs cholinergiques. L'acétylcholine libérée dans le ganglion par les fibres préganglionnaires agit sur des récepteurs de type nicotinique portés par les fibres post-ganglionnaires et l'activation de ces récepteurs produit une bronchoconstriction, bloquée par l'hexaméthonium, inhibiteur spécifique des récepteurs nicotiniques.

L'acétylcholine libérée par la fibre post-ganglionnaire agit sur des récepteurs de type muscarinique, qui sont bloqués par l'atropine et ses dérivés de synthèse. Les techniques de fixation d'un agoniste ou d'un antagoniste marqué par un radioélément, sur les récepteurs muscariniques de membranes pulmonaires isolées ou en autoradiographie, ont montré que les récepteurs sont les plus abondants sur les voies aériennes de gros et de moyen calibre [7].

Chez l'homme, la distribution des récepteurs muscariniques n'est pas connue, mais le faible pouvoir contractant de l'acétylcholine sur des fragments de parenchyme, suggère que ces récepteurs sont probablement peu nombreux au niveau des bronchioles terminales. Les effets intracellulaires des agents muscariniques passent probablement par l'inhibition de l'adénylate cyclase d'une part, et d'autre part par la stimulation de la phospholipase $\mathrm{C}$, une enzyme membranaire qui induit le catabolisme des phospholipides associés au récepteur muscarinique. Adénylate cyclase et phospholipase C sont liées aux récepteurs par des protéines régulatrices, dites Gprotéines, GTP-dépendantes. Les dérivés des phospholipides, comme le diacylglycérol [8], un activateur de la protéine kinase $\mathrm{C}$, responsable de la phosphorylation de différentes protéines, et les inositol phosphates [9] capables de mobiliser le calcium intracellulaire, sont directement impliqués dans la contraction [10] (figure 1). Des récepteurs muscariniques sont aussi présents dans les mastocytes et dans les glandes sousmuqueuses, dont la sécrétion et le transfert ionique trans-épithélial sont stimulés par les agonistes cholinergiques.

Différentes sous-classes de récepteurs muscariniques ont été identifiées, grâce au développement d'antagonistes sélectifs comme la pirenzépine, qui inhibe spécifiquement les récepteurs $M 1$. Dans le tube digestif comme dans l'arbre bronchique, les récepteurs M1 sont portés par les extrémités nerveuses et les ganglions, alors que les récepteurs M2 sont présents sur le muscle lisse. Il est possible qu'il existe des récepteurs M1 dans les glandes. Les récepteurs M1 et M2 sont inhibés par l'atropine et ses dérivés. Des récepteurs muscariniques inhibiteurs ont récemment été identifiés [11]. Le blocage de ces récepteurs par l'atropine pourrait paradoxalement 
contribuer à une exacerbation de la bronchoconstriction, par accroissement de la libération d'acétylcholine, et à une plus grande difficulté au blocage des récepteurs M2 (figure 2).

\section{Neuromédiateurs adrénergiques}

Innervation. L'innervation sympathique provient des six segments thoraciques de la moelle épinière, et les fibres pré- et postganglionnaires font relais dans les ganglions cervicaux médian et inférieur et dans les quatre ganglions thoraciques supérieurs. Les fibres post-ganglionnaires pénètrent dans le poumon au niveau du hile, et forment un plexus dense avec les fibres cholinergiques. A l'opposé du système cho- linergique, les fibres adrénergiques sont peu nombreuses dans le poumon et le contenu en noradrénaline du poumon humain est très inférieur à celui du cœur et des vaisseaux systémiques. Les fibres adrénergiques, identifiables par immunocytochimie ou par utilisation d'un anticorps dirigé contre la dopamine $\beta$-hydroxylase, spécifique des nerfs adrénergiques, sont visibles autour des glandes sousmuqueuses et des artères bronchiques, et très rarement au contact du muscle lisse des voies aériennes. La microscopie électronique montre la présence de petits granules à centre dense dans les voies nerveuses adrénergiques, beaucoup moins abondants que les granules de type cholinergique. La stimulation par champ électrique du muscle lisse in vitro dans le bain musculaire, (qui agit en stimulant les voies nerveuses présentes dans la préparation conduisant ensuite à la contraction du muscle lisse), produit une bronchoconstriction suivie de relaxation. Chez l'homme, cette relaxation n'est pas inhibée par le propranolol (un $\beta$-bloquant non sélectif), mais elle est inhibée par la tétrodotoxine, confirmant sa nature nerveuse et non pas musculaire. Fonctionnellement, la tyramine, un inhibiteur de la recapture des catécholamines, n'est pas bronchodilatatrice chez l'homme. Ces faits confirment l'absence d'innervation adrénergique du poumon humain [12].

Bien qu'il n'y ait pas d'innervation fonctionnelle directe des voies aériennes, il est possible que les nerfs sympathiques contrôlent
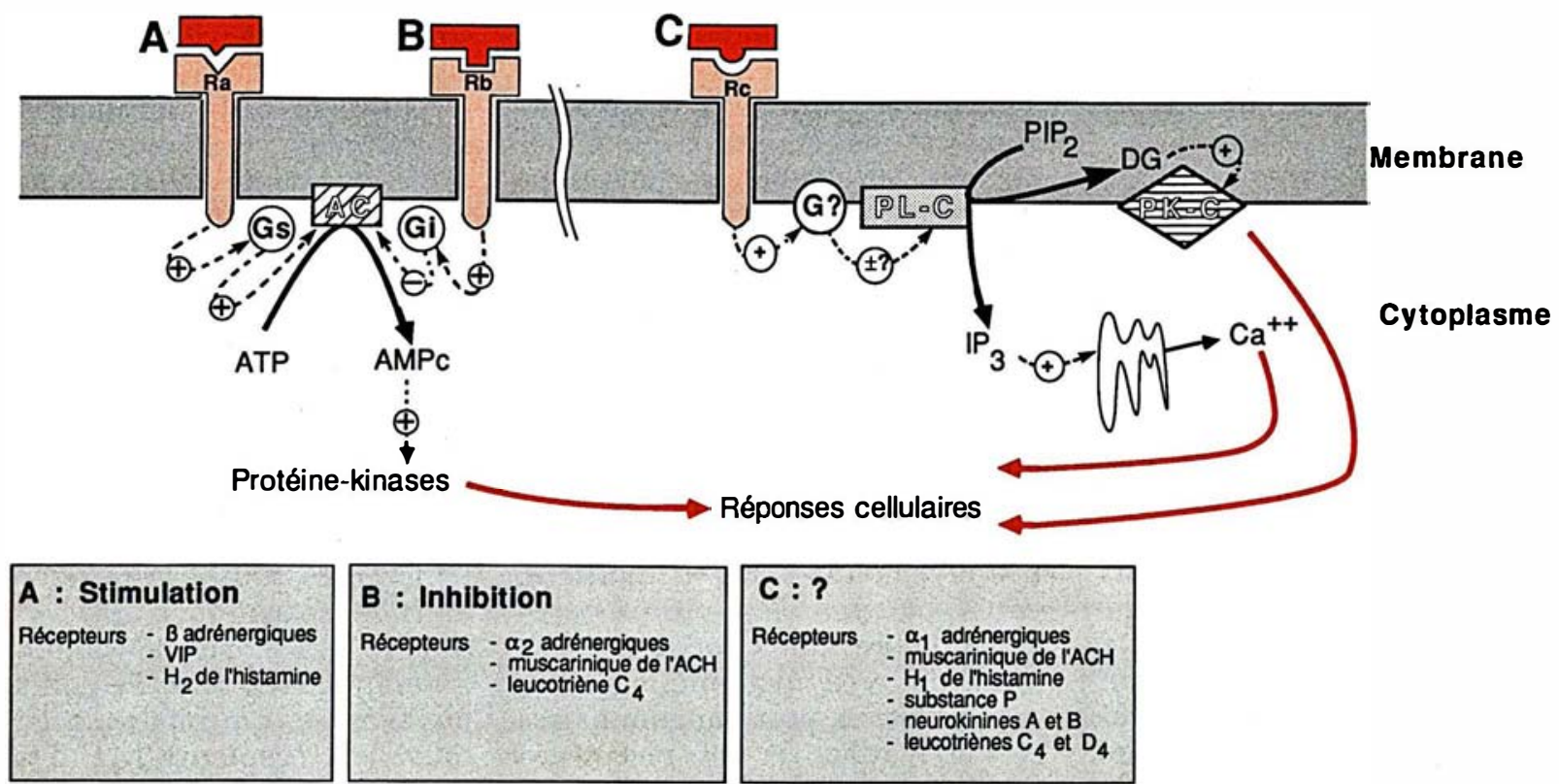

Figure 1. Mode d'action des neuromédiateurs dans les cellules pulmonaires : stimulation et inhibition par différents agonistes des récepteurs membranaires couplés aux enzymes de la face endocellulaire qui jouent le rôle d'effecteurs en déterminant la production de messagers intracellulaires. Les agonistes qui stimulent l'adénylate cyclase (AC), induisent l'accumulation d'AMP cyclique. Ils dilatent le muscle lisse et s'opposent en général à la libération de médiateurs par les cellules inflammatoires. Les agonistes qui stimulent la phospholipase $C$ (PL-C) induisent l'accumulation de diacylglycérol (DG) et d'inositol trisphosphate qui contribuent à l'activation des protéines contractiles et à la mobilisation du calcium intracellulaire. Ces agonistes entraînent la contraction du muscle lisse. II est aussi possible qu'ils inhibent l'adénylate cyclase, et les protéines GTP dépendantes (G) I, qui couplent récepteurs et phospholipase C, pourraient être semblables aux protéines GTP-dépendantes inhibitrices $\left(G_{i}\right)$ de l'adénylate cyclase. $A C H=a c e ́ t y l c h o l i n e$ $P K-C=$ protéine kinase $C$ (d'après $[28 \mathrm{~J})$. Ra, $R b, R c=$ récepteurs des ligands de types $A, B$ et $C$; tracés noirs continus $=$ réactions chimiques $;$ tracés noirs discontinus $=$ effets physiologiques directs $;$ tracés rouges $=$ effets physiologiques globaux. 


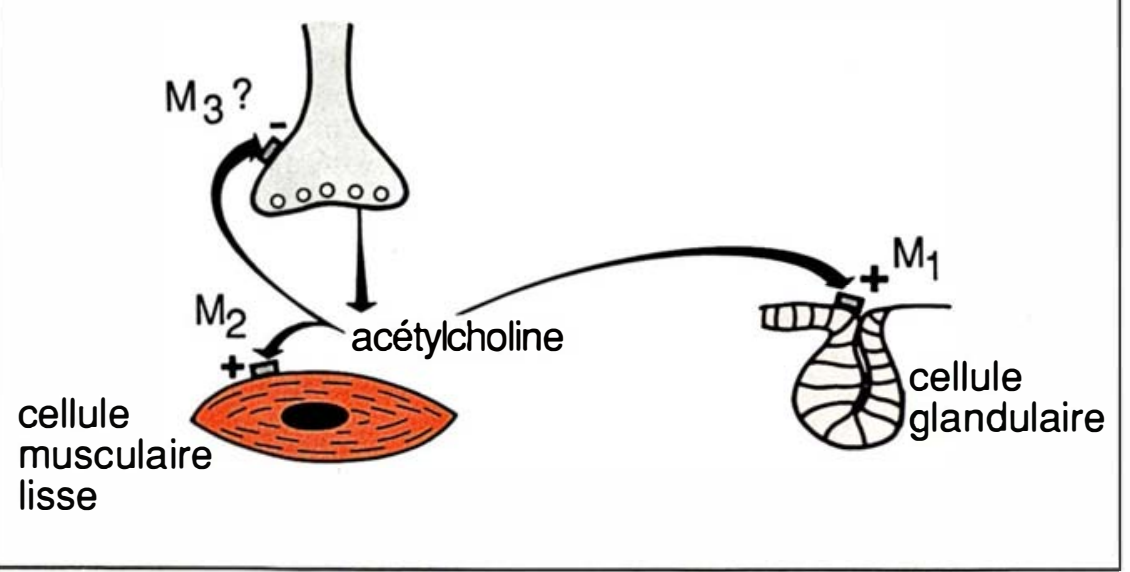

Figure 2. Récepteurs pulmonaires des neuromédiateurs : les récepteurs muscariniques. Le sous-type $M_{2}$ est porté par le muscle lisse. II détermine la contraction; le sous-type $M_{1}$ est de localisation plus incertaine dans le poumon. II est possible qu'il prédomine dans les glandes sous-muqueuses. Récemment a été identifié un autre sous-type $\left(M_{3}\right.$ ?) pré-synaptique. La stimulation de ce récepteur inhibe la libération d'acétylcholine dans la jonction entre fibres pré- et post-ganglionnaires. Un antagoniste comme l'atropine bloque les trois sous-types de récepteurs, et peut conduire à une augmentation de l'influx cholinergique par inhibition des " $M_{3}$ ", alors même qu'il bloque la conséquence de cet accroissement, en inhibant les $M_{2}$ (d'après [29]).

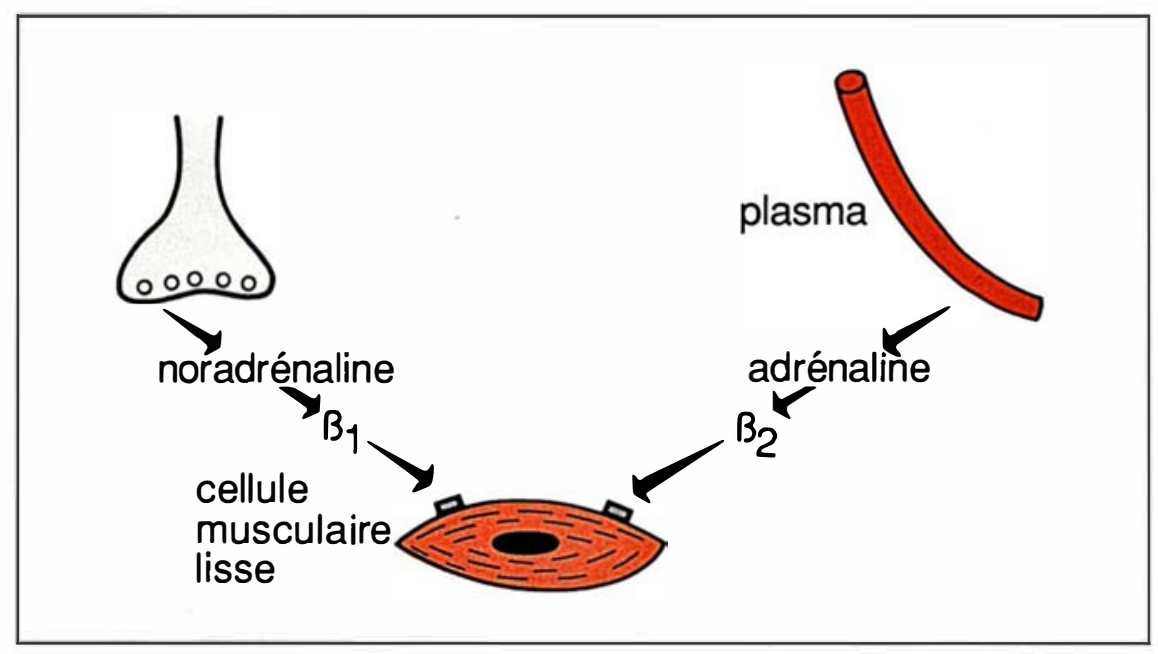

Figure 3. Récepteurs pulmonaires des neuromédiateurs : les récepteurs adrénergiques. Les récepteurs $\beta_{2}$-adrénergiques (stimulés par l'adrénaline circulante) prédominent par rapport aux récepteurs $\beta_{1}$-adrénergiques (stimulés par la noradrénaline des voies de conduction nerveuses). Les deux sous-types sont bronchodilatateurs. La stimulation des récepteurs $\alpha$-adrénergiques a peu $d^{\prime}$ effet sur le muscle lisse des voies aériennes, mais les agonistes $\alpha$ adrénergiques sont de puissants vasoconstricteurs.

$\mathrm{m} / \mathrm{s} n^{\circ} 1$ vol. 4, janvier 88 indirectement le tonus bronchique, puisque la stimulation directe d'un tronc nerveux sympathique produit une bronchodilatation, bien qu'il n'y ait pas de ramification nerveuse adrénergique intrapulmonaire. Ce contrôle peut s'exercer soit par le biais d'une modulation du tonus cholinergique [13], soit parce que la noradrénaline libérée au niveau vasculaire diffuse jusqu'au muscle lisse des voies aériennes [1]. Dans certaines espèces, l'effet bronchodilatateur des agonistes $\beta$-adrénergiques dépend du tonus vagal pré-existant. La modulation adrénergique du système cholinergique pourrait s'exercer par l'intermédiaire des récepteurs adrénergiques présents dans le ganglion parasympathique. Chez le sujet normal les $\beta$ bloquants ne sont pas bronchoconstricteurs, mais ils peuvent précipiter un bronchospasme chez l'asthmatique, suggérant qu'il existe un contrôle adrénergique accru du tonus bronchique chez l'asthmatique.

Il est également possible que les catécholamines circulantes provenant des surrénales participent au contrôle adrénergique du tonus bronchique. L'adrénaline surrénalienne est un bronchodilatateur puissant chez le sujet normal et chez l'asthmatique, aux concentrations physiologiques, et protège contre la bronchoconstriction induite par l'histamine. A l'inverse les $\beta$-bloquants potentialisent la réponse bronchoconstrictrice à l'histamine et aux antigènes chez l'animal sensibilisé, et il est probable que ces effets sont dus à un blocage des catécholamines circulantes. Le rythme diurne de sécrétion des catécholamines est normal chez l'asthmatique. A l'exercice, il existe un retard à l'augmentation de la sécrétion d'adrénaline, mais pas d'anomalie quantitative de la production de ce médiateur. Ces faits sont en accord avec la survenue possible de bronchospasme lors du début d'un exercice chez l'asthmatique, suivie par une récupération de la fonction respiratoire après l'échauffement. 
Les récepteurs adrénergiques (figure 3 ).

(a) Les $\boldsymbol{\beta}$-adrénocepteurs pulmonaires sont très abondants dans toutes les espèces. Ils sont localisés au niveau de différentes cellules pulmonaires. La densité [15] des $\beta$-adrénocepteurs sur le muscle lisse augmente avec la diminution du diamètre des voies aériennes, en accord avec le fait que les $\beta$-agonistes sont capables de réduire la contraction dans les bronches et le parenchyme pulmonaire. Il existe également des $\beta$-adrénocepteurs dans les glandes muqueuses plus que séreuses, et sur l'épithélium (16]. Les cellules de Clara sont porteuses de $\beta$-adrénocepteurs, et lors de leur stimulation par des $\beta$-agonistes, sécrètent un lipide dans les voies aériennes, dont la fonction pourrait être d'empêcher la fermeture de cellesci. Les pneumocytes de type 2, qui sécrètent le surfactant y sont stimulés par les $\beta$-agonistes. Il existe des $\beta$-adrénocepteurs sur les mastocytes pulmonaires, et les $\beta$ agonistes empêchent la libération de médiateurs mastocytaires in vitro et in vivo. Les $\beta$-agonistes réduisent ou empêchent l'induction par l'histamine de la fuite plasmatique hors des vaisseaux. Il n'est pas prouvé que les $\beta$ agonistes diminuent l'œème des voies aériennes, bien qu'ils empêchent son instauration. Les $\beta$ agonistes réduisent aussi la neurotransmission cholinergique au niveau ganglionnaire.

(b) Les $\alpha$-adrénocepteurs sont présents dans le poumon de nombreuses espèces, y compris chez l'homme, mais sont peu abondants $[7,15]$. Fonctionnellement, leur stimulation ne conduit à la contraction du muscle lisse qu'en présence d'un blocage des $\beta$ adrénocepteurs, ou lorsque le muscle lisse est pré-traité avec un médiateur, comme l'histamine ou la sérotonine. Toutefois ces médiateurs ne modifient pas le nombre et l'affinité des $\alpha$-récepteurs, suggérant qu'un mécanisme postrécepteur est impliqué dans l'accroissement de la sensibilité aux agents adrénergiques, en présence de ces médiateurs. Chez l'homme les récepteurs $\alpha$-adrénergiques augmentent avec l'âge et dans certaines affections (asthme, bronchite chronique), bien que leur rôle dans ces affections ne soient pas clairement défini. Les $\alpha$ agonistes sont de puissants contractants du muscle lisse vasculaire. Ils stimulent la sécrétion glandulaire de mucus et déclenchent la libération de médiateurs par le mastocyte ; ils inhibent la conduction nerveuse au niveau ganglionnaire, suggérant ainsi la présence d' $\alpha$ récepteurs à ces différents niveaux. Il existe des $\alpha$-récepteurs, qui sont post-synaptiques et déterminent la contraction, et des $\alpha_{2}$-récepteurs qui sont présynaptiques et inhibent la libération de noradrénaline par les terminaisons nerveuses synaptiques [17].

\section{Neuromédiateurs non cholinergiques non adrénergiques}

L'existence d'un système nerveux autonome non-cholinergique nonadrénergique est connue depuis longtemps au niveau du tube digestif, et puisque les voies aériennes ont la même origine embryologique, il a été suggéré que le même système pourrait aussi exister dans les voies aériennes.

La stimulation électrique des voies aériennes produit une bronchoconstriction qui est bloquée partiellement par l'atropine, suivie d'une bronchodilatation qui n'est pas inhibée par le propranolol, ce qui évoque respectivement l'existence de neuromédiateurs stimulateurs non cholinergiques et inhibiteurs non adrénergiques. Il a été initialement proposé que les médiateurs du système non cholinergique non adrénergique étaient les purines, qui peuvent reproduire certains de ses effets, mais certains peptides sont actuellement de meilleurs candidats au rôle de neuromédiateurs du NANC (neuromédiateurs non cholinergiques, non adrénergiques) dans les voies aériennes.

Système inhibiteur non adrénergique.

(a) Innervation. Il existe des nerfs inhibiteurs non adrénergiques dans 
différentes espèces dont l'homme. Chez l'homme, le système inhibiteur non adrénergique est le seul système relaxant puisque il n'y a pas d'innervation adrénergique fonctionnelle. Le NANC non adrénergique innerve chez l'homme, les voies aériennes petites et larges. La bronchodilatation due au NANC prédomine sur les voies aériennes larges. La bronchodilatation produite est intense et de longue durée. Les nerfs du NANC non adrénergique sont pré-ganglionnaires (l'effet de la stimulation du NANC est inhibé par les substances bloquantes ganglionnaires) et post-ganglionnaires (la stimulation du muscle lisse par champ électrique in vitro reproduit ses effets). Histologiquement les nerfs du NANC sont reconnaissables à la présence de vésicules larges à centre dense, les granules de type "p ", qui contiennent des peptides comme la substance $\mathrm{P}$ ou le VIP (vasoactive intestinal peptide), identifiables par immunocytochimie [18]. Les récepteurs des voies afférentes sont mal connus ; la stimulation mécanique du larynx induit une bronchodilatation réflexe du NANC chez le chat. Chez l'homme, la stimulation du larynx produit aussi une bronchodilatation, mais la voie réflexe complète n'a pas été explorée. Le NANC non adrénergique module également la sécrétion de mucus chez l'animal [18, 19].

(b) Les médiateurs du NANC inhibiteur non-adrénergique. Il est peu probable que l'ATP, l'adénosine ou les purines soient les médiateurs du NANC, car, si l'ATP et l'adénosine relâchent le muscle lisse pré-contracté, leurs antagonistes n'inhibent pas cet effet et le dypiridamole, un inhibiteur de la recapture des purines, ne le potentialise pas. La présence des vésicules " $\mathrm{p}$ " dans le poumon humain à côté des vésicules caractéristiques des nerfs cholinergiques et adrénergiques, suggère que les peptides régulateurs peuvent jouer un rôle dans la régulation du tonus des voies aériennes.

Parmi les peptides isolés dans les voies aériennes, seuls le VIP et $\mathrm{m} / \mathrm{s} n^{\circ} 1$ vol. 4 , janvier 88 deux peptides voisins le PHI (peptide histidine isoleucine) et le PHM (peptide histidine methionine) relâchent les voies aériennes, et il est très probable que VIP est au moins l'un des transmetteurs du NANC non adrénergique dans les voies aériennes. Le VIP est un peptide de 28 acides aminés qui a été identifié dans les terminaisons des neurones qui innervent le muscle lisse, les glandes sousmuqueuses et les vaisseaux bronchiques et pulmonaires, et aussi les ganglions et les extrémités nerveuses pré-ganglionnaires [20]. Les fibres VIP-ergiques identifiées en immunocytochimie prédominent au niveau des voies aériennes de gros calibre. Le VIP est libéré dans le bain musculaire lors de la stimulation électrique, témoignant de son origine nerveuse. Il est possible que le VIP coexiste dans les mêmes extrémités nerveuses que l'acétylcholine et soit un cotransmetteur de l'acétylcholine. Le VIP pourrait moduler la libération d'acétylcholine par un effet pré-jonctionnel. Le VIP n'est libéré que lors d'une stimulation vagale intense et pourrait contrer l'effet de l'acétylcholine par un mécanisme d'antagonisme fonctionnel. Il est aussi possible que le VIP module l'apport sanguin au muscle lisse lors de la contraction cholinergique.

Le VIP produit, in vitro, une bronchodilatation prolongée 50 fois plus puissante que la bronchodilatation à l' isoprénaline dans la bronche humaine. Elle n'est pas bloquée par l'atropine, le propranolol et l'indométhacine, suggérant un effet direct sur le muscle lisse passant par des récepteurs propres. Chez l'animal, le VIP prévient ou rend réversible la bronchoconstriction à différents agonistes. Chez l'homme, le VIP inhalé est un bronchodilatateur peu puissant. Le VIP infusé n'a pas d'effet sur le tonus des voies aériennes à des doses où l'effet cardiovasculaire est important. Le faible effet du VIP infusé in vivo peut s'expliquer par le faible accès du VIP au muscle lisse (il s'agit d'une molécule de poids moléculaire élevé), par la destruction enzymatique ou par le fait que les effets cardiovasculaires limitent la dose à administrer. La préincubation du muscle lisse des voies aériennes avec du VIP diminue l'effet de doses ultérieures de VIP (désensibilisation des récepteurs) et l'effet relaxateur du NANC dans certaines espèces, témoignant que le VIP est un neuromédiateur. Dans d'autres modèles animaux (trachée de cobaye), la pré-incubation avec le VIP ne modifie pas l'effet bronchodilatateur du NANC, et ceci suggère que d'autres peptides que le VIP peuvent être libérés par les nerfs inhibiteurs non adrénergiques. Ainsi, le rôle du VIP comme médiateur de la contraction semble certain, mais ne pourra être confirmé que lors du développement et de l'emploi d'antagonistes spécifiques.

Il existe des nerfs VIP-ergiques au contact des glandes sousmuqueuses, et le VIP est un stimulant de la sécrétion de mucus par les glandes trachéales chez différents animaux, mais il inhibe la sécrétion de macromolécules par les bronches humaines. Le VIP est un puissant stimulant du transport du chlore à travers l'épithélium. Le VIP au total augmente le transport muco-ciliaire. La présence de fibres nerveuses contenant des vésicules larges et denses dans les ganglions parasympathiques suggère qu'il peut exister une régulation $\mathrm{du}$ système cholinergique par le VIP. (c) Les récepteurs au VIP. Les récepteurs au VIP sont identifiables sur l'épithélium, les glandes sous-muqueuses et le muscle lisse, ainsi que le montre l'autoradiographie utilisant du VIP marqué à l'iode 125. L'effet du VIP passe par l'AMP cyclique [21].

Système excitate $r$ non cholinergique.

(a) Innervation. La stimulation électrique des grosses bronches des voies aériennes et parfois de la trachée de cobaye provoque une contraction qui n'est pas inhibée par l'atropine. Cette réponse est reproduite par la substance $P$ et inhibée par les antagonistes de celle-ci, suggérant que la subs- 


\section{RÉFÉRENCES}

22. Lundberg JM, Saria A, Brodin E, Rusell $S$, Folkers R. A substance $P$ antagonist inhibits vagally induced increase in vascular permeability and bronchial smooth muscle contraction in the guinea-pig. Proc Natl Acad Sci USA 1983 ; 80 : 1120-4.

23. Theodorsson-Norheim E, Hua X, Brodin E, Lundberg JM. Capsaicin treatment decreases tissue levels of neurokinin A-like immunoreactivity in the guinea-pig. Acto Physiol Scand $1985 ; 124: 129-31$.

24. Grandordy BM, Frossard N, Rhoden KJ, Barnes PJ. Tachykinin-induced contraction is modulated by epithelium in guinea-pig airways. Proc Natl Acad Si USA 1987 (sous presse).

25. Lundberg JM, Anders FC, Hua X, Hökfelt T, Fischer JA. Coexistence of substance $P$ and calcitonin gene-related peptide-like immunoreactivity in sensory nerves in relation to cardiovascular and bronchoconstrictor effects of capsaicin. Eur J Pharmacol 1985; 108 : 315-9.

26. Sheppard MN, Polak JM, Allen JM, Bloom SR. Neuropeptide tyrosine (NYP) : a newly discovered peptide is present in the mammalian respiratory tract. Thorax 1984 ; 39 : 326-30.

27. Ekblad E, Hakanson R, Sundler F, Wahlestedt C. Galanin : neuromodulatory and direct contractile effects on smooth muscle preparations. Br J Pharmacol 1985 ; 86 : 241-6.

28. Grandordy BM. A role for phospholipid in bronchial reactivity? Thorax, 1987 (sous presse). nes PJ (en préparation). tance $\mathrm{P}$ est un neurotransmetteur $\mathrm{du}$ système excitateur noncholinergique. Dans les bronches humaines, le système excitateur non cholinergique paraît plus difficile à mettre en évidence.

(b) Les médiateurs du système excitateur non cholinergique. La substance $P$ est synthétisée dans le ganglion nodal et transportée vers l'extrémité distale de la voie afférente, d'où elle peut être libérée expérimentalement par stimulation antidromique (conduction nerveuse se faisant dans le sens contraire de la stimulation habituelle), et in vivo chez l'animal par administration de capsaïcine.

Chez l'homme l'inhalation de capsaïcine induit une toux et une bronchoconstriction minimes et passagères. La perfusion de substance $\mathrm{P}$ a des effets cardiovasculaires importants mais peu d'effets bronchiques. Les effets cardiovasculaires limitent la dose qu'il est possible d'administrer, et peuvent être responsables de bronchodilatation réflexe par réduction du tonus vagal, qui suit la bronchoconstriction initiale due à la substance P. Par inhalation, la substance $\mathrm{P}$ a également peu d'effet, mais il est possible qu'elle soit dégradée par les enzymes épithéliales [22-24].

In vitro, la substance $P$ contracte le muscle lisse, stimule la sécrétion glandulaire muqueuse et induit un œdème muqueux par accroissement de la perméabilité capillaire. Le traitement néonatal de l'animal par la capsaïcine (qui empêche le développement normal des fibres contenant la substance $\mathrm{P}$ ), prévient l'extravasation plasmatique produite par différents stimulants mécaniques et chimiques, suggérant que celle-ci est déterminée par la substance $P$. La substance $P$ facilite la libération d'histamine par le mastocyte péritonéal, et il est possible qu'elle exerce les mêmes effets sur les cellules inflammatoires des voies aériennes. Il est également possible que la substance $P$ soit chimiotactique pour les polynucléaires, contribuant à l'apparition ou au maintien de la réponse inflammatoire.

Le calcitonin gene related peptide
(CGRP) est un peptide localisé aux voies nerveuses afférentes. C'est probablement un cotransmetteur de la substance P. Il a été extrait des voies aériennes. In vitro, le CGRP provenant du poumon humain est un des plus puissants agents contractants du muscle lisse bronchique humain. Il agit vraisemblablement par l'intermédiaire de récepteurs spécifiques [25].

D'autres peptides, dont le rôle n'est pas certain, ont été identifiés dans le poumon. Le neuropeptide $Y$ est localisé dans les vaisseaux pulmonaires, dont il est un puissant agent contractant. La distribution des nerfs contenant le neuropeptide $Y$ est semblable à celle des nerfs sympathiques, $e_{t}$ il est possible que le neuropeptide $Y$ soit un cotransmetteur de l'adrénaline. La galanine est localisée dans les nerfs moteurs du système respiratoire ; elle est peut-être colocalisée avec l'acétylcholine et le VIP. Sa fonction, ainsi que celle du gastrin-releasing peptide, demeure inconnue [26, 27].

(c) Les récepteurs à la substance $P$. La substance $P$ agit par l'intermédiaire de récepteurs spécifiques, identifiables par fixation de substance $\mathrm{P}$ marquée à l'iode 125 à des membranes de tissu pulmonaire ou par autoradiographie. La sensibilité à la substance $\mathrm{P}$ et à certains de ses dérivés (les tachykinines) varie avec les tissus, suggérant l'existence de différents types de récepteurs aux tachykinines. Récemment, deux autres tachykinines ont été identifiées par immunocytochimie, dans le poumon de mammifère, la neurokinine $A$ et la neurokinine $B$. La neurokinine A est un agent contractant plus puissant que la neurokinine $B$, elle-même plus puissante que la substance $P$. A l'inverse, la fuite plasmatique vasculaire induite par la substance $P$ est plus importante que celle induite par la neurokinine A et la neurokinine $\mathrm{B}$. Les récepteurs du muscle lisse sont dits de type NK2 $(S P . E)$, ceux du système vasculaire de type NK1 (SP.P).

Les récepteurs au CGRP et aux autres peptides n'ont pas encore 
été caractérisés dans le poumon et il se pourrait que plusieurs de ces systèmes ne soient pas fonctionnels : il apparaît maintenant bien établi que le tonus cholinergique n'est pas augmenté chez l'asthmatique par rapport au sujet normal ainsi que le montre la réactivité normale de ces sujets aux anticholinergiques de synthèse. Il est possible que l'augmentation apparente de la réactivité aux agonistes cholinergiques, à l'histamine, à différents irritants spécifiques ou non spécifiques in vivo, soit due à un accès facilité de ces agonistes au muscle lisse, par exemple du fait d'anomalies constitutives ou acquises de la barrière épithéliale. Certaines cellules, comme les éosinophiles, anormalement accumulées dans l'épithélium des voies aériennes peuvent libérer localement des protéines toxiques qui fragilisent la barrière épithéliale. Il est possible aussi que les médiateurs formés par les cellules épithéliales ou les cellules présentes dans l'épithélium, comme les mastocytes, soient libérés en quantité particulièrement importante chez l'asthmatique. La présence en quantité anormale d'irritants et de médiateurs dans les voies aériennes pourrait favoriser la stimulation des fibres $\mathrm{C}$, la libération de peptides par les extrémités sensitives de ces fibres et les réflexes à point de départ locaux.

Il est vraisemblable que dans les années à venir, d'autres peptides ou d'autres médiateurs seront identifiés dans les voies aériennes. Les peptides déjà connus peuvent agir comme médiateurs directs de l'interaction entre le nerf et l'organe cible, mais il est probable qu'ils exercent aussi d'autres fonctions et sont capables en particulier de moduler la transmission nerveuse au niveau ganglionnaire ou pré-synaptique dans l'organe cible. Les techniques permettant de mesurer la production de médiateurs par les extrémités nerveuses, ainsi que le développement d'antagonistes spécifiques de ces peptides permettront certainement de mieux étudier ces interactions

\section{Summary}

Airway hyper-reactivity in asthma and other diseases may be the consequence of a dysfunctioning of autonomic nervous system. The adrenergic, the cholinergic and the non-adrenergic non-cholinergic branches of the autonomic nervous system can be identified in airways of various species including man, by functional, radioimmunological, histochemical and binding studies. Neuromediators are specific of the various branches, but more than one mediator can be identified in each branch. Acetylcholine in the cholinergic system and noradrenaline in the adrenergic system are released in the efferent nervous pathway, and at the neuro-muscular junction are responsible for modifications of smooth muscle tension, via the activation of specific receptors. Glands and vessels are also innervated by cholinergic and adrenergic systems. Tachykinins, in the non-adrenergic non-cholinergic nervous system, are released in the afferent branch, possibly at the level of irritant receptors in airway mucosa, leading to various effects such as smooth muscle contraction, attraction of inflammatory cells, mucus secretion and vasodilatation. Other peptides in airway nonadrenergic non-cholinergic system, such as VIP (vasointestinal peptide) might have a relaxing effect on smooth muscle. Specific receptors to peptides have been identified in lung. The mechanisms of cellular activation following stimulation of receptors are now better understood, and involve activation of membrane enzymes, as adenylate cyclase and phospholipase C. In the future, development of new drugs (selective antagonits and blockers of intracellular messengers) might help to better understand hyperreactivity and provide new treatment for asthma. 